\title{
TRAPLINE: a standardized and automated pipeline for RNA sequencing data analysis, evaluation and annotation
}

\author{
Markus Wolfien ${ }^{1 *}$ (D), Christian Rimmbach², Ulf Schmitz, ${ }^{3,6}$ Julia Jeannine Jung ${ }^{2}$, Stefan Krebs ${ }^{4}$, Gustav Steinhoff ${ }^{2}$, \\ Robert David ${ }^{2^{*}}$ and Olaf Wolkenhauer ${ }^{1,5}$
}

\begin{abstract}
Background: Technical advances in Next Generation Sequencing (NGS) provide a means to acquire deeper insights into cellular functions. The lack of standardized and automated methodologies poses a challenge for the analysis and interpretation of RNA sequencing data. We critically compare and evaluate state-of-the-art bioinformatics approaches and present a workflow that integrates the best performing data analysis, data evaluation and annotation methods in a Transparent, Reproducible and Automated PipeLINE (TRAPLINE) for RNA sequencing data processing (suitable for Illumina, SOLiD and Solexa).

Results: Comparative transcriptomics analyses with TRAPLINE result in a set of differentially expressed genes, their corresponding protein-protein interactions, splice variants, promoter activity, predicted miRNA-target interactions and files for single nucleotide polymorphism (SNP) calling. The obtained results are combined into a single file for downstream analysis such as network construction. We demonstrate the value of the proposed pipeline by characterizing the transcriptome of our recently described stem cell derived antibiotic selected cardiac bodies ('aCaBs').

Conclusion: TRAPLINE supports NGS-based research by providing a workflow that requires no bioinformatics skills, decreases the processing time of the analysis and works in the cloud. The pipeline is implemented in the biomedical research platform Galaxy and is freely accessible via www.sbi.uni-rostock.de/RNAseqTRAPLINE or the specific Galaxy manual page (https://usegalaxy.org/u/mwolfien/p/trapline—manual).
\end{abstract}

Keywords: RNA sequencing, NGS data processing, Data evaluation, Bioinformatics workflow, Galaxy, TRAPLINE, Stem cells

\section{Background}

In comparison to other high-throughput methods, Next Generation Sequencing (NGS) technologies enable genome-wide investigations of various phenomena, including single-nucleotide polymorphisms, epigenetic events, copy number variants, differential expression, and alternative splicing [1]. RNA sequencing (RNAseq)

\footnotetext{
* Correspondence: markus.wolfien@uni-rostock.de; robert.david@med.unirostock.de

Markus Wolfien, Christian Rimmbach and UIf Schmitz are first author Robert David and Olaf Wolkenhauer are senior author

'Department of Systems Biology and Bioinformatics, University of Rostock, 18057 Rostock, Germany

${ }^{2}$ Reference und Translation Center for Cardiac Stem Cell Therapy (RTC),

University of Rostock, Rostock 18057, Germany

Full list of author information is available at the end of the article
}

uses the NGS technology for discovering novel RNA sequences, and quantifying all transcripts in a cell [2,3]. Like genome tiling arrays, an RNAseq experiment can capture evidence for yet unannotated genes and isoforms. The utility of RNAseq to uncover new transcripts is well documented [3-8]. Several laboratories have provided evidence that cDNA library preparation and RNA sequencing sets are technically well reproducible and in contrast to microarrays RNAseq offers a broader dynamic range, which makes this platform more sensitive in the detection of transcripts with low abundance [9].

The steady increase of publications involving RNAseq experiments generated a need for statistical and computational tools to analyze the data. Basically, all RNAseq 
analyses involve the following tasks: pre-processing, quality control, read mapping and further analyses like differential expression (DE) analysis, single nucleotide polymorphism (SNP) analysis or gene isoform and splicing variant detection. However, the availability of tools following a standardized analysis protocol are limited [10].

A number of software packages and pipelines have already been introduced to deal with these tasks. These software packages are mainly based on programming languages like $C$, Python or $R$ and require advanced expertise in programming or computer science for proper implementation and use or they do not provide advanced analytical tools like gene network inference methods, miRNA-target predictions and/or the integration of protein-protein interactions [11-16]. Additionally, the possibility of discovering alternatively spliced genes or promoter activity would be desirable. Furthermore, there is no common RNAseq data analysis strategy, despite the obvious need for such a standardized pipeline [17]. The increased dependence on computational approaches in life sciences has revealed grave concerns about the accessibility and reproducibility of the computed results [5]. Galaxy is a free web-based platform for omics research that addresses the following needs $[18,19]$ :

- Accessibility: Galaxy enables users to perform integrative omics analyses by providing a unified, webbased interface for obtaining omics data and applying computational tools to analyze these data. Learning a programming language or the implementation details is not necessary.

- Reproducibility: Galaxy produces metadata about every possible analysis step and automatically tracks descriptive information about datasets, tools, and parameter values to ensure reproducibility. User annotations and tagging is possible at each step of the pipeline.

- Transparency: Galaxy includes a web based framework for sharing models including datasets, histories, workflows and repositories. It also allows users to communicate and discuss their experimental results in an online forum.

\section{Implementation}

Using Galaxy, we developed a comprehensive, Transparent, Reproducible and Automated analysis PipeLINE, named TRAPLINE, for RNAseq data processing (optimized for Illumina FASTQ reads, but also suitable for other sequencing platforms like SOLiD or Solexa), evaluation and prediction. The predictions are based on modules which are able to identify protein-protein interactions, miRNA targets and alternatively splicing variants or promoter enriched sites. A schematic representation of the analysis pipeline is illustrated in Fig. 1. TRAPLINE can be accessed via the published Galaxy page of TRAPLINE (https://usegalaxy.org/u/mwolfien/p/trapline-manual) or via www.sbi.uni-rostock.de/RNAseqTRAPLINE.

TRAPLINE implements the following tools and resources: (i) FASTQ quality trimmer, FASTXclipper and FastQC for pre-processing and quality control, (ii) TopHat2 for read mapping, (iii) Picard Toolkit for read correction and SNP identification, (iv) Cufflinks2/Cuffdiff2 for DE analysis, splicing and promoter testing (v) the Database for Annotation, Visualization and Integrated Discovery (DAVID) for gene annotation and functional classification, (vi) miRanda for miRNA target prediction, (vii) BioGRID for protein-protein interactions and, finally, a compiling module for ready to use network construction files. For detailed instructions regarding the usage of TRAPLINE please see the manual in the Additional file 1.

\section{Results}

To show the effectiveness of our automated pipeline, we exemplarily applied TRAPLINE to RNAseq data generated from our recently described antibiotic selected cardiac bodies ("aCaBs"), which are highly pure clusters of mouse embryonic stem cell (mESC) derived cardiomyocytes generated via Myh6 promoter based antibiotic selection plus a standardized differentiation protocol (Additional file 2: Figure S1) [20, 21]. Their RNA expression profiles were compared to control embryoid bodies (EBs) derived from the same cell line without administration of the antibiotic.

\section{TRAPLINE includes state-of-the-art quality control processes}

TRAPLINE analyses RNAseq reads obtained from Illumina, SOLiD and Solexa platforms with the help of "FASTQ Groomer" [22] that converts the specific formats as a first step. In the following pre-processing step, adapter sequences, which have been added to the $5^{\prime}$ and 3 ' ends of the cDNA fragments during the sample preparation phase, are being clipped (no influence towards other platforms). In the Illumina sequencing procedure, sequences are extended on both ends by - 62 nucleotide long adapters that may influence the results of the subsequent analysis [23]. These adapters are only used during the Illumina bridge amplification procedure to immobilize the cDNA transcripts. In TRAPLINE we implemented the tool "FASTXClipper" (http://hannonlab.cshl.edu/fastx_toolkit/index.html) for this purpose.

It is necessary to discriminate sequencing errors from biological variation by using quality scores (Q) [24]. Therefore, in the last pre-processing step uncalled and wrongly called bases are removed (Quality Trimming). Standard approaches rely on the associated quality 


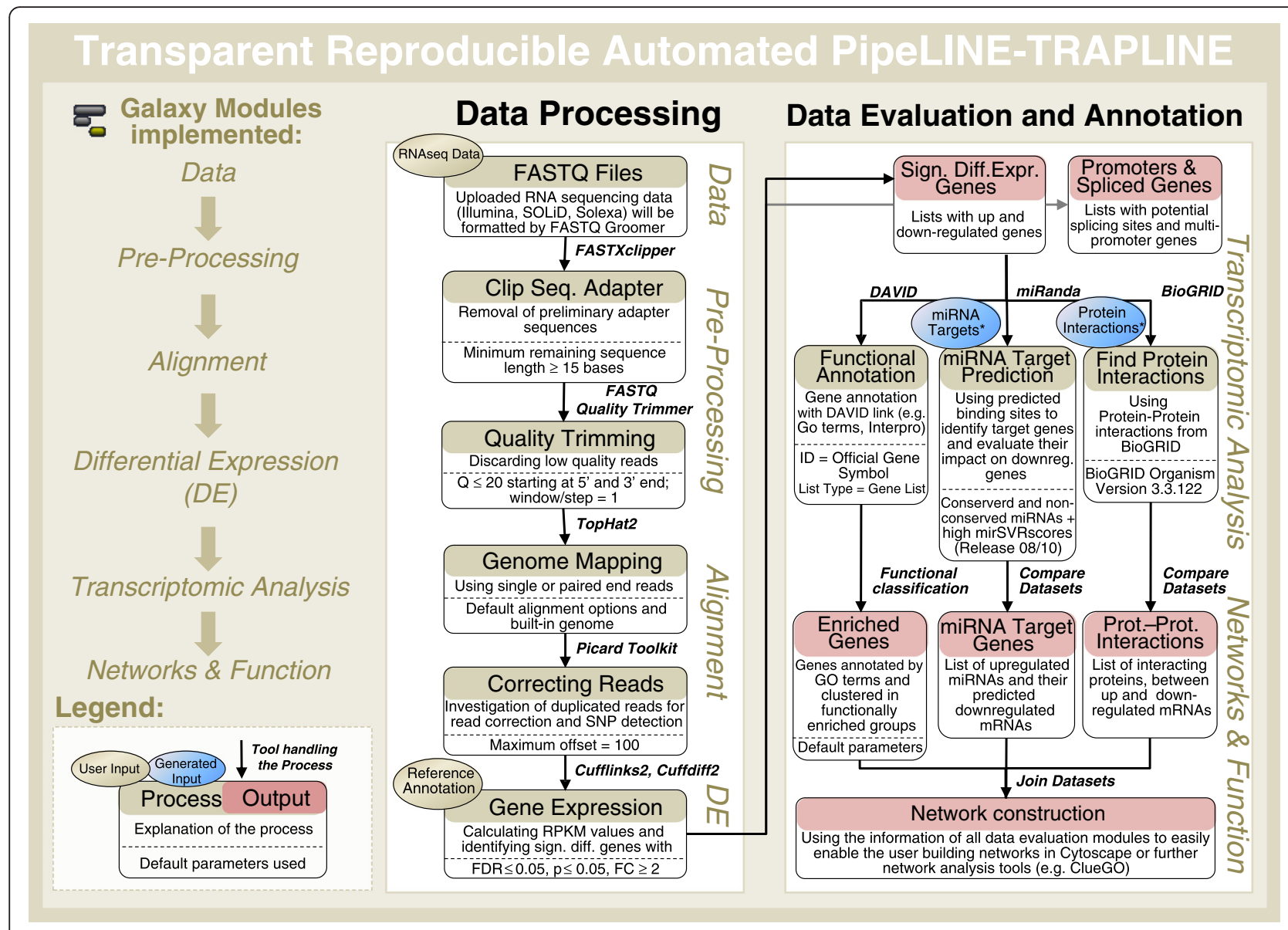

Fig. 1 Scheme to illustrate TRAPLINE'S RNA sequencing analyses modules. * The target files are provided as Galaxy histories - miRNA Targets (usegalaxy.org/u/ mwolfien/h/trapline-mirna-targets-input) and Protein-Protein Interactions (usegalaxy.org/u/mwolfien/h/trapline-protein-protein-interaction-input)

scores to retain the read, or a portion of it, if the score is above a predefined threshold [22]. As suggested by Mbandi et al. [24] we excluded reads with a score $\mathrm{Q}<20$ to ensure reliable genome mapping results. For the purpose of discarding low quality reads, we implemented the widely used tool "FASTQ Quality Trimmer" which returns a quality control report for each dataset that was analyzed. The effects achieved by quality trimming our data are shown in Additional file 3: Figure S2.

We compared the fraction of mapped reads with and without applying data pre-processing (Fig. 2a). Our observations confirm the findings of Chen et al. [25], who demonstrated the necessity of applying the described pre-processing steps in a read-mapping benchmark.

TopHat2 - the most accurate alignment tool in Galaxy

To select the most suitable read alignment tool, we analyzed the overall mapped transcript coverage on the genome (accuracy) of the most commonly used alignment tools, which are based on the exon first approach. Figure $2 \mathrm{~b}$ shows the results of our comparison between BWA [26],
Bowtie [27], Bowtie2 [28], and TopHat2 [29], and their average accuracy in the mapping of six different datasets. The overall alignment accuracy of the mapped reads to the reference genome is between $70 \%$ and $85 \%$. Bowtie 2 and TopHat2, that share a similar algorithm, produce a significantly higher accuracy in comparison to the BWA and Bowtie alignment tools (based on a significance level $\alpha=$ 0.05). In our case, the Bowtie 2 alignment algorithm was able to map in average 2.5 million more reads to the genome than the BWA/Bowtie algorithm (total amount reads: 24-26 million). Our observations are consistent with the results of Kim et al. [30], who found that TopHat2 generates more accurate alignments than competing tools, using fewer computational resources. Because of the significantly superior mapping accuracy of TopHat2, in contrast to Bowtie/BWA, and the additional functionality to find splice junctions and promoter regions, we decided to include TopHat2 into TRAPLINE. The outputs of TopHat 2 are BAM files which contain the aligned reads to the reference genome and text files summarizing the accuracies of the mapped reads for each FASTQ file. 
A

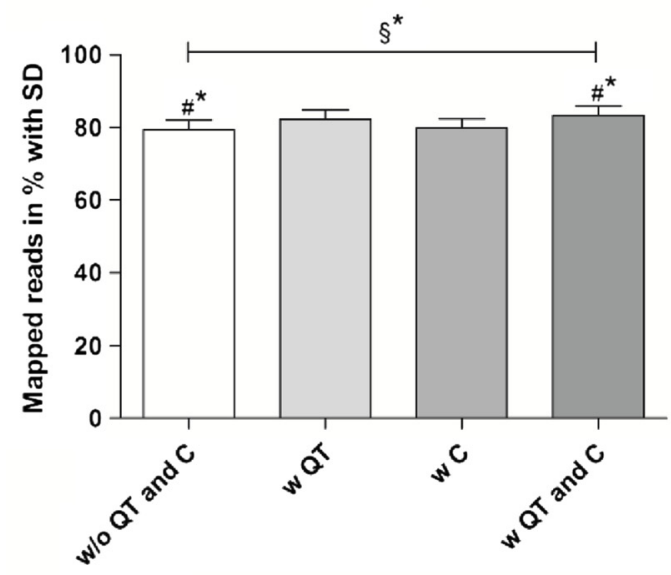

C

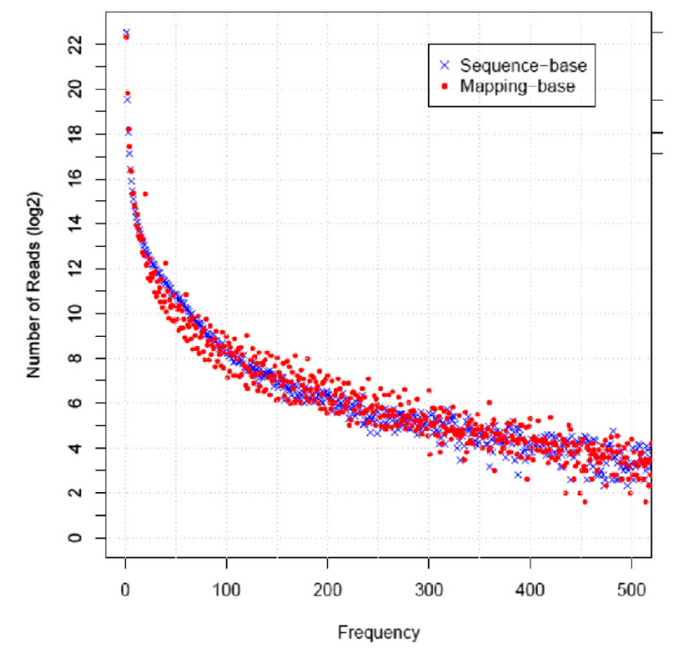

E

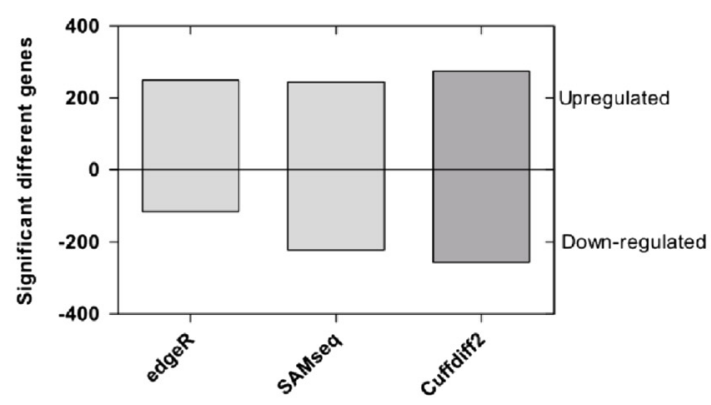

B

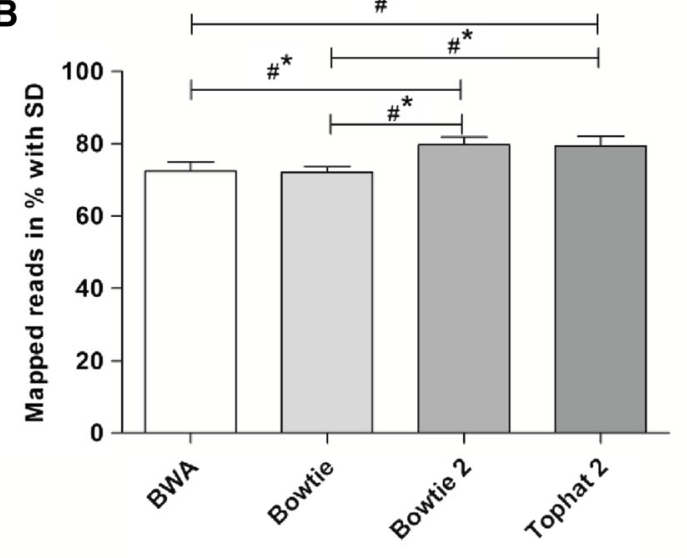

D

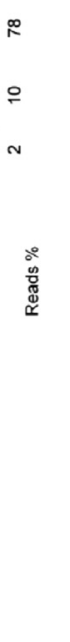

F

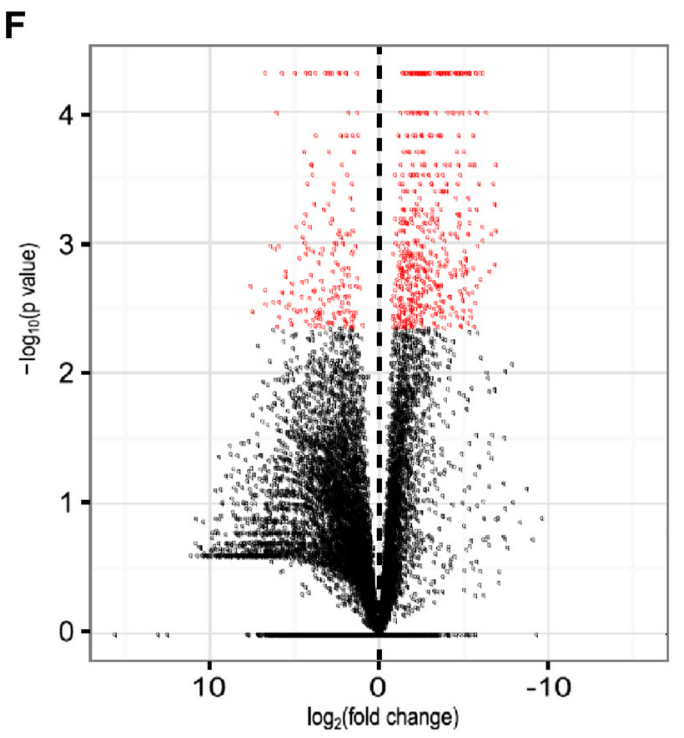

Fig. 2 (See legend on next page.) 


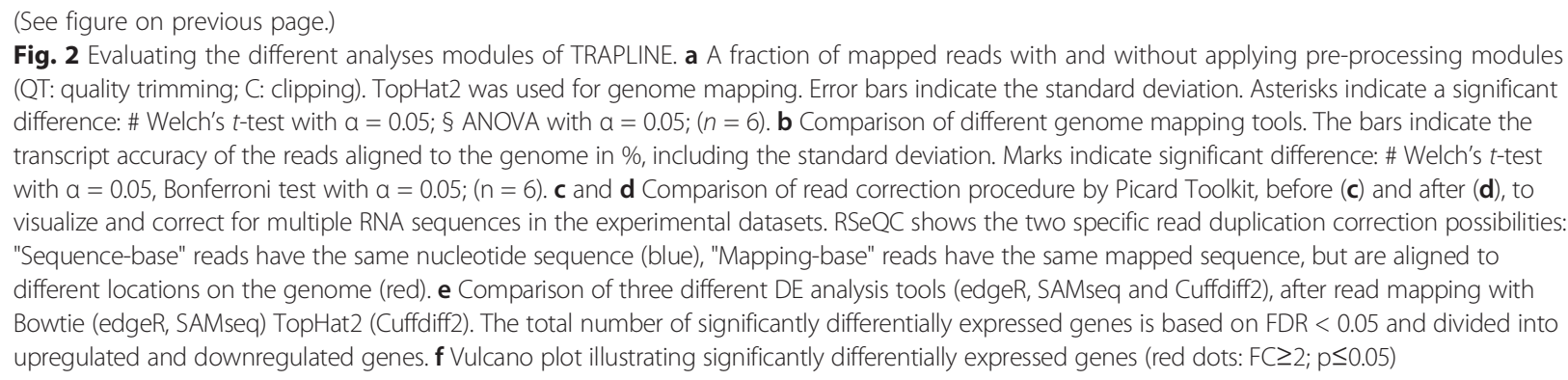

\section{Correction of reads is necessary for SNP detection}

The presence of duplicates is a major issue in single/ paired short reads from NGS platforms. PCR amplification is one of the major sources of duplicates, which are usually introduced during sequencing library amplification [31]. These duplicates might have a serious impact on research applications, especially towards SNP detection, because they can confound the expression data of a particular gene and, therefore, are usually removed [32]. A popular tool for this task is "MarkDuplicates" from the Picard toolkit (https://github.com/broadinstitute/picard), which finds the 5' coordinates and mapping orientations of each read pair and removes them. During this procedure, the tool considers all clipping that has taken place as well as any gaps or jumps in the alignment. To investigate the influence of the duplicate removing step with Picard tools, we determined the read duplication rates and the number of reads mapped to the same location using the "RSeQC" Python module [33]. The results are visualized in Fig. 2c and d. RSeQC uses two strategies to determine read duplication rates: (i) sequence based (blue dots), which means reads with identical sequences are regarded as duplicated reads; (ii) mapping based (red dots) which expresses reads that are mapped to exactly the same genomic location. A comparison of both figures clearly shows an elevation of the mappingbase. The red dots are refined in Fig. 2d in contrast to Fig. 2c, meaning that there were many aligned reads with a similar mapping sequence but with a different location on the genome, which was corrected by "MarkDuplicates". The corrected bam-files can be further investigated by a SNP calling analysis software such as GATK [34] or CRISP [35].

\section{Cuffdiff2 adds value to the standard DE analysis}

The different DE analysis methods are based on (i) negative binomial models, such as edgeR, DEseq, baySeq, (ii) non-parametric approaches, such as SAMseq, NOIseq and (iii) transcript-based detection methods, such as Cuffdiff2 and EBSeq [36]. We compared the performances of the most widely used DE tools from each group, which are Cuffdiff2 [37], edgeR [38] and SAMseq [39], to show how they compare and to underline the
DE analysis efficiency of TRAPLINE. Prior to the analysis reads were mapped to the reference genome with different methods (Bowtie for edgeR/SAMseq and TopHat2 for Cuffdiff2), because each tool has different data input requirements. Figure 2e shows only slight differences between the applied DE methods. All tools nearly identified the same amount of genes as significantly upregulated among in aCaBs compared to EBs ( 250), however different amounts of genes were classified as downregulated. In general, the statistical approaches used by edgeR and SAMseq are more liberal in defining significant differences than the Cuffdiff2 algorithm [17]. In agreement with our results, these widely used methods have recently been compared by several research groups [17, 36, 40, 41]. Cuffdiff2 estimates expression at transcript-level resolution and controls the variability and read mapping ambiguity by using a beta negative binomial model for fragment counts [37]. Furthermore, the tool enhances the comparability between experiments, because it uses the derived "reads per kilobase per million" (RPKM) mapped reads metric [3] which normalizes for both gene size (more reads or fragments can be mapped to larger genes) and the total number of reads or fragments (per million mapped). Seyednasrollah et al. [17] stated Cuffdiff 2 as the most conservative DE method with the lowest false positive rate. Therefore, we included Cuffdiff2 for RNAseq DE analysis in TRAPLINE to retrieve precise results with highly significant genes.

As default setting Cuffdiff 2 considers genes as significant for $\mathrm{p} \leq 0.05$, and a fold change (FC) higher than two. Another reason to integrate Cuffdiff2 into TRAPLINE is the possibility to determine differential splicing events and to perform differential promoter testing [42]. This possibility qualifies the pipeline to investigate for genes with two or more splice variants and genes producing two or more distinct primary transcripts (multi-promoter genes). Multiple splice and promoter isoforms are often co-expressed in a given tissue [3].

We have performed a performance test between TRAPLINE and other tools. A summary of the ratio of mapped reads, discarded reads and significantly differentially 
expressed genes obtained with the indicated tools is shown in Additional file 4: Table S1.

\section{Gene annotation, miRNA target prediction and protein- protein interactions with TRAPLINE}

Additionally, we included three data annotation and prediction steps into TRAPLINE. First, filtering modules were implemented to scan the list of differentially expressed genes and extract sets of upregulated and downregulated genes. Additionally, users receive a link to DAVID [43] to evaluate the functional influences of the significantly upregulated/downregulated transcripts within their data. In general, DAVID finds Gene Ontology terms (GO terms), signaling pathways (based on databases like Panther, KEGG, Biocarta, etc.) or protein domains (e.g. based on InterPro) that are predominantly associated with lists of genes (e.g. from a DE analysis). Moreover, DAVID performs a functional annotation clustering analysis that groups these terms into functionally related clusters which gives the user a first and quick insight into the biological impact of the discovered differences [44]. Second, TRAPLINE includes modules for miRNA target prediction that use significantly upregulated and downregulated miRNAs and automatically spot possible targets among the downregulated or upregulated mRNAs in the analyzed datasets. For this purpose we provide formatted text files of conserved and non-conserved miRNAs and their predicted targets for different species (human, mouse, rat, fruitfly and nematode), based on the latest version of the microRNA.org database (release 2010; [45]). The files can be obtained via a Galaxy history and have to be uploaded as TRAPLINE "miRNA targets" input. Third, we implemented a module which is able to identify verified interactions between proteins of significantly upregulated and downregulated mRNAs. The protein-protein interactions are based on data from peer-reviewed publications deposited in the BioGRID database (release 3.3.122; [46]). Similar to the miRNA targets, we provide protein-protein interactions from five different species (human, mouse, rat, fruitfly and nematode) in the form of Galaxy history files and will continuously extend the species.

\section{Identifying transcriptomic differences of $\mathrm{EBs}$ and $\mathrm{aCaBs}$}

A step by step description on how to use TRAPLINE is provided in the Supplementary Material section. In summary, users upload FASTQ files from a RNAseq experiment, select the reference genome for the species under investigation and run the pipeline to obtain the significantly differentially expressed transcripts. Optionally, one can upload the provided miRNA target and protein interaction files to use the full potential of TRAPLINE. Exemplarily, we applied the developed pipeline on RNAseq data from our murine ESC derived aCaBs [21] in comparison to control EBs.

We uploaded in total six datasets (as fastqsanger files), the murine reference annotation (as gtf or gff3 that can be obtained from http://geneontology.org/page/reference-genome-annotation-project), the mm9 miRNA targets file (from the provided Galaxy history), the $\mathrm{mm} 9$ protein interactions file (also from the history) and ran TRAPLINE with the default parameter settings. After a processing time of $\sim 10 \mathrm{~h}$ we retrieved the results. We found $\sim 550$ significantly differentially expressed transcripts, 260 of which were upregulated. The volcano plot shown in Fig. $2 \mathrm{f}$ illustrates the results of the DE analysis. It shows the ratio of the significantly differentially expressed genes (red) against the non-significant genes (black). At this point one might want to lower the cutoff of the p-value to obtain less reads marked as significant, which is easily possible by tuning the corresponding Cuffdiff2 parameter. However, we took the 260 upregulated genes as input for the subsequent functional classification analysis with DAVID, which revealed several annotation clusters. The first three annotation clusters contain 160 genes in total and suggest a biological impact on the cytoskeleton, actin and the contractile fibers (Additional file 5: Table S2). Based on the annotated biological processes described by GO terms, we created a network to show the links and significance of each GO term using the Cytoscape application ClueGo [47]. The network is shown in Fig. 3 and illustrates the 260 upregulated genes that are associated with enriched biological processes. The distribution of significant biological processes is illustrated in Additional file 6: Figure S3. These genes could be a starting point for subsequent analyses.

We also predicted miRNA interactions of downregulated mRNAs. Their associated GO terms suggest an impact on cardiac cell differentiation. Exemplarily, we show the significantly upregulated miRNA "mmu-mir369" with 5.522 predicted targets which include 57 genes that are downregulated in aCaBs (Additional file 7: Table S3). These 57 genes were functionally classified by DAVID and reveal a high probability to affect the cell cycle and to support cell differentiation. Among these genes is "Atp1a2" which is known to negatively regulate heart function [48]. Furthermore, we analyzed the $\sim 550$ significantly differentially expressed mRNAs and identified $\sim 230$ verified protein interactions, 10 splice variants and 12 multi promoter regions (Additional file 8: Table S4).

\section{Discussion}

We developed TRAPLINE for RNAseq data analysis to link differentially expressed transcripts to the corresponding phenotypic changes and biological phenomena. There exist other tools such as the Bioconductor packages edgeR and DEseq [49], that are with no doubt 


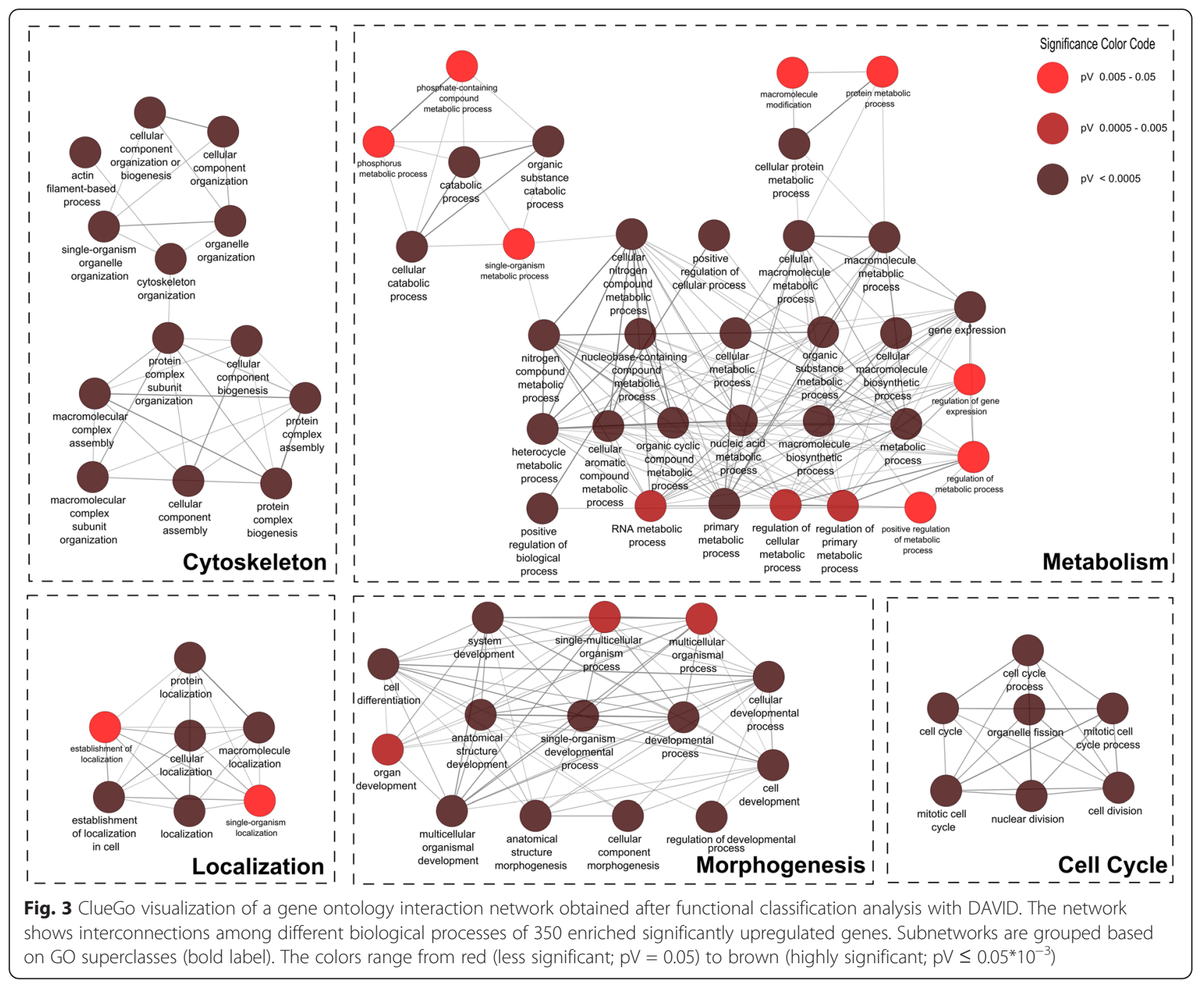

valuable resources to support the analysis of NGS data. Our pipeline, however, includes pre-processing and genome mapping modules and, is furthermore, easily applicable. TRAPLINE mainly addresses researchers with limited or no programming skills e.g. in $R$ or Python. We are confident that the graphical user interface of TRAPLINE, which is implemented in the Galaxy platform, greatly supports the accessibility of our RNAseq data analysis pipeline to users with no computational background. Furthermore, we have carefully selected a set of best performing interconnected modules that evade compatibility or file formatting issues. The entire RNAseq data analysis workflow can thus be performed in one go without losing the flexibility that experienced users appreciate when being enabled to adjust module parameters to their own needs. Different to other automated workflows like $\mathrm{MeV}$, Chipster, RobiNA or Grape our pipeline is additionally predicting spliced variants, enriched promoter sites, miRNA targets and protein-protein interactions to enable users getting a comprehensive insight to the analyzed samples [11-14]. There are several other Galaxy pipelines available online, for example the widely used Oqtans workbench [15]. Oqtans is a collection of tools without a pre-defined pipeline. In contrast, our work for the first time introduces an automated Galaxy workflow that includes detailed data analysis and data annotation on a public Galaxy server. TRAPLINE is using all benefits of Galaxy and is independent of computational resources (i.e. no need for high performance computers). Researchers can access and share their data and the results worldwide via the internet, however Galaxy also offers private accounts and the possibility to install a local Galaxy instance on a private machine, which is beneficial in case of limited internet connectivity. Moreover, Galaxy enables a synchronous work, e.g. four read mapping tasks at a time are possible. In our case study the time for the analysis was reduced to $10 \mathrm{~h}$ in comparison to a desktop $\mathrm{PC}$ requiring $24 \mathrm{~h}$ (Additional file 9: Table S5). Additionally, to accomplish a transparent computing speed analysis, we performed a comparison between a standard TRAPLINE run 
at the public Galaxy instance and a local desktop PC based on a randomly selected publicly available SRA dataset (BioProject:PRJNA292442; SRA study: SRP062238) [50].

The implementation of Cuffdiff2 for detecting differentially expressed genes enhances the comparability between various RNAseq experiments, because the method is accompanied by RPKM normalization [37]. Nevertheless, it has to be considered that the RPKM value for a gene from a deep library may have more statistical meaning than an equivalent value from a more shallow library [51]

It is known that spatial biases along the genome exist, resulting in a non-uniform coverage of expressed transcripts [3]. Especially when using Cufflinks, it has been shown that DE analysis attempting to correct for differences in gene length have the tendency of introducing a bias in the per gene variances, in particular for lowly expressed genes [52]. These spatial biases hinder comparisons between genomic regions and will therefore adversely affect any analysis where such a comparison is integrated. To overcome this problem the current version of Cufflinks2 has an integrated bias correction algorithm [53]. In our investigated datasets there was no need for a bias correction, therefore, we turned this feature off (Additional file 10: Figure S4). It can be reimported manually by setting the respective Cufflinks parameter.

With respect to the biological reliability of the results, the number of our above described 550 significantly differentially expressed genes could be further reduced based on p-value and fold change adjustments. Please be aware that the performance of our pipeline was evaluated based on the Illumina sequencing platform that was used to generate the experimental data. Additionally, it is possible to apply different multiple testing correction method like Bonferroni or Benjamini-Hochberg [54]. Using the same parameters, all three applied methods deliver similar results for differentially expressed genes. With the default parameter values, the pipeline also considers genes which are only slightly up or downregulated $(|F C| \geq 2)$. The gene annotation clustering approach enables enrichment in information and a pointer to the biological relevance of the apparently large number of differentially expressed genes. Gene Ontology terms and especially the gene set enrichment analysis performed by DAVID are established methods for gaining first insights into phenotype variations between the tested experimental conditions [43]. Interestingly, the first three enriched GO term clusters in our case study relate to biological processes concerning the cytoskeleton and actin regulation which are two core factors of cardiomyocytes and thus provide a proof of principle for our pipeline (Additional file 5: Table S2).

After successful DE analysis, there are several possibilities for further data evaluation and characterization of the transcripts. As we already showed, the GO terms and differentially expressed mRNAs can be visualized as interaction networks using Cytoscape. miRanda predictions have the largest relative overlap with other miRNA prediction algorithms/tools [55], which is why we chose to include miRanda predictions into TRAPLINE in the first place. A SNP analysis with respective tools can also be done by simply using the SNP output of TRAPLINE. Additionally, a co-expression network analysis could be performed to identify co-expressed mRNAs that are simultaneously dis-regulated [56].

\section{Conclusion}

Taken together, our proposed pipeline includes all relevant RNA sequencing data processing modules, is easily applicable, and needs no time consuming installation processes. TRAPLINE guides researchers through the NGS data analysis process in a transparent and automated state-of-the-art pipeline. Experimentalists will be able to analyze their data on their own without learning programming skills or advanced computational knowledge. The data can be accessed worldwide and can optionally be shared among researchers. Gaining quickly in-depth insights into the biology underlying the investigated data, our work for the first time introduces an automated Galaxy workflow including detailed data processing, data evaluation and annotation modules (www.sbi.uni-rostock.de/RNAseqTRAPLINE).

\section{Availability and requirements \\ Project name: TRAPLINE}

Project home page: https://usegalaxy.org/u/mwolfien/ $\mathrm{p} /$ trapline-manual

Operating system(s): Platform independent

License: Galaxy Web Portal Service Agreement (https:// usegalaxy.org/static/terms.html)

\section{Materials and methods \\ Cell culture and aCaB-Generation}

Murine ES cell lines described previously [57] were grown in high glucose DMEM with stable glutamine (GIBCO) containing $10 \%$ FBS Superior (Biochrom), $100 \mu \mathrm{M}$ nonessential amino acids (GIBCO), 1 \% Penicillin/Streptamycin (GIBCO) and $100 \mu \mathrm{M} \beta$-Mercaptoethanol (Sigma) in presence of $1000 \mathrm{U} / \mathrm{mL}$ of Leukemia inhibitory factor (LIF, Millipore). Differentiation of aCaBs was performed in hanging drop culture for two days using 1000 cells as starting material for one EB in Iscove's basal medium (Biochrom) containing $10 \%$ FBS (Biochrom), $100 \mu \mathrm{M}$ non-essential amino acids (GIBCO), $1 \%$ Penicillin/Streptamycin (GIBCO) and $450 \mu \mathrm{M}$ 1-Thioglycerol. For additional 4 days, the cells were differentiated in suspension culture, and at day 6 of differentiation consistently 15 EBs were seeded on one well of a 24-well-plate. Antibiotic 
selection with $400 \mu \mathrm{g} / \mathrm{mL}$ G418 (Biochrom) was initiated at day 8 post seeding. 4 days thereafter, aCaBs were isolated via treatment with $6000 \mathrm{U} / \mathrm{mL}$ Collagenase IV (GIBCO) for $30 \mathrm{~min}$. To obtain single cells for subsequent experiments, the bodies were further dissociated with $100 \%$ Accutase (Affimetrix) for $15 \mathrm{~min}$. To ensure successful generation of aCaBs, potential mycoplasma contamination was routinely controlled twice a week using the PCR based MycoSPY kit system (Biontex).

\section{RNA-Sequencing}

For library generation and sequencing, cultured adherent cells were drained from the culture medium, washed and directly lysed by addition of lysis buffer. $1 \mathrm{ul}$ of this lysate was used for cDNA Synthesis and amplification with the SMARTer kit (Clontech, Mountain View CA, USA) according to the manufacturer's instructions. In brief, cDNA synthesis was initiated by annealing a polyA-specific primer and adding a reverse transcriptase with terminal transferase activity. The newly synthesized first strand cDNA is then tailed first with a homopolymer stretch by terminal transferase and then with a specific amplification tag by template switching. The resulting double-tagged cDNA was amplified by PCR, fragmented by sonication (Bioruptor, Diagenode, Liege Belgium; 25 cycles $30 \mathrm{~s}$ on/30 s off) and converted to barcoded Illumina sequencing libraries using the NEBnext Ultra DNA library preparation kit (New England Biolabs, Ipswich MA, USA). After PCR enrichment the libraries were purified with AmpureXP magnetic beads (Beckman-Coulter, Brea CA, USA) and quantified on a Bioanalyzer 2100 (Agilent, Santa Clara CA, USA). Libraries were pooled at equimolar amounts and sequenced on an Illumina GenomeAnalyzer IIx in single-read mode with a read-length of 78 nucleotides and a depth of 21 million to 32 million raw reads per replicate.

\section{Additional files}

Additional file 1: TRAPLINE manual: Step by Step instructions for the usage. (DOC $35 \mathrm{~kb})$

Additional file 2: Figure S1. Flowchart for $\mathrm{aCaB}$ Generation. Cartoon is displaying sequential steps for the generation of aCaBs, combining Myh6promoter selection and an additional cell-dissociation step [21]. (TIF $90 \mathrm{~kb}$ )

Additional file 3: Figure S2. Visualization for RNA transcript quality control and comparison of per base quality score $\mathrm{Q}$. The images are taken before (A) and after (B) quality trimming procedure (removes reads with $Q \leq 20$ ) to estimate the effect of trimming. The quality score $Q$ is plotted to the read position by using the FastQC package in Galaxy (http://www.bioinformatics.babraham.ac.uk/projects/fastac). The color indicates the quality of the read: "red" low quality, "orange" median quality, "green" good quality. Red line expresses the mean of the measured values (yellow boxes are inter-quartile range) and the blue line represents the mean quality. (ZIP $81 \mathrm{~kb}$ )

Additional file 4: Table S1. Performance comparison of TRAPLINE vs other tools. (DOC $28 \mathrm{~kb}$ )
Additional file 5: Table S2. Example for DAVID functional gene annotation clustering of significantly differentially expressed genes from $\mathrm{aCaBs}$ and EBs. (DOC $48 \mathrm{~kb}$ )

Additional file 6: Table S3. Exemplarily we show a result of a miRNA target prediction analysis of TRAPLINE. (TIF $84 \mathrm{~kb}$ )

Additional file 7: Figure S3. Pie chart illustrating enriched biological processes of upregulated genes in the $\mathrm{aCaB}$ derived cardiomyocytes. The chart presents the enriched GO superclasses. (DOC $26 \mathrm{~kb}$ )

Additional file 8: Table S4. Exemplarily results of protein-protein interaction prediction, splice variants and multi promoter regions. (DOC $37 \mathrm{~kb}$ )

Additional file 9: Table S5. Benchmarking results of TRAPLINE performed on a public Galaxy server and on a local desktop PC (based on computing speed). (DOC $31 \mathrm{~kb}$ )

Additional file 10: Figure S4. A comparison of experiments without (A) and with (B) bias correction performed with the help of Cufflinks2. The dots represent the dependency of the log ratio of two FPKM values (M) and their mean average (A). The MA plot is a common method to investigate the biases of datasets [53]. (PPTX $236 \mathrm{~kb}$ )

\section{Competing interests}

The authors declare that they have no competing interests.

\section{Authors' contributions}

MW, CR, US, JJJ, GS and RD developed the idea. MW developed the workflow and did the Galaxy implementation. CR and JJ designed and conducted the experiments. SK performed the RNA sequencing. MW analyzed the experimental data. CR, US, RD helped with the analysis and biological interpretation of the data. OW provided the general framework for bioinformatics study. MW, US, RD, OW contributed in drafting the manuscript, revising it critically and approved the final version. MW, CR and US share first authorships. RD and OW are joint senior authors on this work. All authors read and approved the final manuscript.

\section{Acknowledgements}

This work has been funded by the Federal Ministry of Education and Research Germany (FKZ 0312138A, FKZ 316159 and FKZ 02NUK043C) and the State Mecklenburg-Western Pomerania with EU Structural Funds (ESF/IV-WM-B340030/10 and ESF/V-BM-B35-0010/12), by the DFG (DA 1296-1), the German Heart Foundation (F/01/12), by the FORUN Program of Rostock University Medical Centre (889001) and the EU funded CaSyM project (grant agreement \#305033).

\section{Author details}

'Department of Systems Biology and Bioinformatics, University of Rostock, 18057 Rostock, Germany. ${ }^{2}$ Reference und Translation Center for Cardiac Stem Cell Therapy (RTC), University of Rostock, Rostock 18057, Germany. ${ }^{3}$ Gene \& Stem Cell Therapy Program, Centenary Institute, 2050 Camperdown, Australia. ${ }^{4}$ Gene Center Munich, LMU Munich, 81377 Munich, Germany. ${ }^{5}$ Stellenbosch Institute of Advanced Study (STIAS), Wallenberg Research Centre at Stellenbosch University, 7602 Stellenbosch, South Africa. ${ }^{6}$ Sydney Medical School, University of Sydney, Sydney, NSW 2006, Australia.

Received: 5 September 2015 Accepted: 22 December 2015 Published online: 06 January 2016

References

1. Hayden EC. Genome sequencing: the third generation. Nature. 2009; 457(7231):768-9.

2. Morozova O, Hirst M, Marra MA. Applications of New Sequencing Technologies for Transcriptome Analysis. Annu Rev Genom Hum G. 2009;10: 135-51.

3. Mortazavi A, Williams BA, Mccue K, Schaeffer L, Wold B. Mapping and quantifying mammalian transcriptomes by RNA-Seq. Nat Methods. 2008;5(7): $621-8$.

4. Hu Y, Wang K, He XP, Chiang DY, Prins JF, Liu JZ. A probabilistic framework for aligning paired-end RNA-seq data. Bioinformatics. 2010;26(16):1950-7.

5. Pepke S, Wold B, Mortazavi A. Computation for ChIP-seq and RNA-seo studies. Nat Methods. 2009;6(11):S22-32. 
6. Ramskold D, Wang ET, Burge CB, Sandberg R. An Abundance of Ubiquitously Expressed Genes Revealed by Tissue Transcriptome Sequence Data. Plos Computational Biology. 2009;5(12):e1000598.

7. Wilhelm BT, Landry JR. RNA-Seq-quantitative measurement of expression through massively parallel RNA-sequencing. Methods. 2009; 48(3):249-57.

8. Wilhelm BT, Marguerat S, Goodhead I, Bahler J. Defining transcribed regions using RNA-seq. Nat Protoc. 2010;5(2):255-66.

9. Marioni JC, Mason CE, Mane SM, Stephens M, Gilad Y. RNA-seq: An assessment of technical reproducibility and comparison with gene expression arrays. Genome Res. 2008;18(9):1509-17.

10. Wang Z, Gerstein M, Snyder M. RNA-Seq: a revolutionary tool for transcriptomics. Nat Rev Genet. 2009;10(1):57-63.

11. Howe EA, Sinha R, Schlauch D, Quackenbush J. RNA-Seq analysis in MeV. Bioinformatics. 2011:27(22):3209-10

12. Kallio MA, Tuimala JT, Hupponen T, Klemela P, Gentile M, Scheinin I, et al. Chipster: user-friendly analysis software for microarray and other highthroughput data. BMC Genomics. 2011;12:507.

13. Knowles DG, Roder M, Merkel A, Guigo R. Grape RNA-Seq analysis pipeline environment. Bioinformatics. 2013;29(5):614-21.

14. Lohse M, Bolger AM, Nagel A, Fernie AR, Lunn JE, Stitt M, et al. RobiNA: a user-friendly, integrated software solution for RNA-Seq-based transcriptomics. Nucleic Acids Res. 2012:40(Web Server issue):W622-7.

15. Sreedharan VT, Schultheiss SJ, Jean G, Kahles A, Bohnert R, Drewe P, et al. Ogtans: the RNA-seq workbench in the cloud for complete and reproducible quantitative transcriptome analysis. Bioinformatics. 2014;30(9):1300-1.

16. Kalari KR, Nair AA, Bhavsar JD, O'Brien DR, Davila Jl, Bockol MA, et al. MAP-RSeq: Mayo Analysis Pipeline for RNA sequencing. BMC Bioinformatics. 2014;15.

17. Seyednasrollah F, Laiho A, Elo LL. Comparison of software packages for detecting differential expression in RNA-seq studies. Brief Bioinform. 2013; 16(1):59-70.

18. Blankenberg $\mathrm{D}$, Hillman-Jackson J. Analysis of next-generation sequencing data using Galaxy. Methods Mol Biol. 2014;1150:21-43.

19. Goecks J, Nekrutenko A, Taylor J, Team G. Galaxy: a comprehensive approach for supporting accessible, reproducible, and transparent computational research in the life sciences. Genome Biology. 2010, 11(8).

20. Rimmbach C, Jung JJ, David R. Generation of Murine Cardiac Pacemaker Cell Aggregates Based on ES-Cell-Programming in Combination with Myh6Promoter-Selection. J Vis Exp. 2015

21. Jung JJ, Husse B, Rimmbach C, Krebs S, Stieber J, Steinhoff G, et al. Programming and isolation of highly pure physiologically and pharmacologically functional sinus-nodal bodies from pluripotent stem cells. Stem Cell Reports. 2014:2(5):592-605.

22. Blankenberg D, Gordon A, Von Kuster G, Coraor N, Taylor J, Nekrutenko A. Manipulation of FASTQ data with Galaxy. Bioinformatics. 2010;26(14): $1783-5$.

23. Del Fabbro C, Scalabrin S, Morgante M, Giorgi FM. An extensive evaluation of read trimming effects on Illumina NGS data analysis. PLOS ONE. 2013; 8(12):e85024.

24. Mbandi SK, Hesse U, Rees DJ, Christoffels A. A glance at quality score: implication for de novo transcriptome reconstruction of Illumina reads. Front Genet. 2014;5:17.

25. Chen C, Khaleel SS, Huang H, Wu CH. Software for pre-processing Illumina next-generation sequencing short read sequences. Source Code Biol Med. 2014;9:8.

26. Li H, Durbin R. Fast and accurate short read alignment with Burrows-Wheeler transform. Bioinformatics. 2009;25(14):1754-60.

27. Langmead B, Trapnell C, Pop M, Salzberg SL. Ultrafast and memory-efficient alignment of short DNA sequences to the human genome. Genome Biol. 2009;10(3):R25

28. Langmead B, Salzberg SL. Fast gapped-read alignment with Bowtie 2. Nat Methods. 2012;9(4):357-9.

29. Trapnell C, Roberts A, Goff L, Pertea G, Kim D, Kelley DR, et al. Differential gene and transcript expression analysis of RNA-seq experiments with TopHat and Cufflinks. Nat Protoc. 2012;7(3):562-78.

30. Kim D, Pertea G, Trapnell C, Pimentel H, Kelley R, Salzberg SL. TopHat2: accurate alignment of transcriptomes in the presence of insertions, deletions and gene fusions. Genome Biol. 2013;14(4):R36.

31. Kozarewa I, Ning ZM, Quail MA, Sanders MJ, Berriman M, Turner D」. Amplification-free Illumina sequencing-library preparation facilitates improved mapping and assembly of (G plus C)-biased genomes. Nat Methods. 2009;6(4):291-5.

32. Xu HB, Luo X, Qian J, Pang XH, Song JY, Qian GR, et al. FastUniq: A Fast De Novo Duplicates Removal Tool for Paired Short Reads. PLoS ONE. 2012;7(12): e52249.

33. Wang L, Wang S, Li W. RSeQC: quality control of RNA-seq experiments. Bioinformatics. 2012;28(16):2184-5.

34. McKenna A, Hanna M, Banks E, Sivachenko A, Cibulskis K, Kernytsky A, et al. The Genome Analysis Toolkit: a MapReduce framework for analyzing next-generation DNA sequencing data. Genome Res. 2010; 20(9):1297-303.

35. Bansal V. A statistical method for the detection of variants from nextgeneration resequencing of DNA pools. Bioinformatics. 2010;26(12): i318-24.

36. Soneson C, Delorenzi M. A comparison of methods for differential expression analysis of RNA-seq data. BMC Bioinformatics. 2013;14.

37. Trapnell C, Hendrickson DG, Sauvageau M, Goff L, Rinn JL, Pachter L. Differential analysis of gene regulation at transcript resolution with RNA-seq. Nat Biotechnol. 2013;31(1):46. -+.

38. Robinson MD, Oshlack A. A scaling normalization method for differential expression analysis of RNA-seq data. Genome Biol. 2010;11(3):R25.

39. Li J, Tibshirani R. Finding consistent patterns: a nonparametric approach for identifying differential expression in RNA-Seq data. Stat Methods Med Res. 2013;22(5):519-36

40. Kvam VM, Liu P, Si Y. A comparison of statistical methods for detecting differentially expressed genes from RNA-seq data. Am J Bot. 2012;99(2): $248-56$.

41. Nookaew I, Papini M, Pornputtapong N, Scalcinati G, Fagerberg L, Uhlen M, et al. A comprehensive comparison of RNA-Seq-based transcriptome analysis from reads to differential gene expression and cross-comparison with microarrays: a case study in Saccharomyces cerevisiae. Nucleic Acids Res. 2012:40(20):10084-97.

42. Trapnell C, Williams BA, Pertea G, Mortazavi A, Kwan G, van Baren MJ, et al. Transcript assembly and quantification by RNA-Seq reveals unannotated transcripts and isoform switching during cell differentiation. Nat Biotechnol. 2010;28(5):511-5.

43. Huang DW, Sherman BT, Lempicki RA. Systematic and integrative analysis of large gene lists using DAVID bioinformatics resources. Nat Protoc. 2009;4(1): 44-57.

44. Huang DW, Sherman BT, Tan Q, Collins JR, Alvord WG, Roayaei J, et al. The DAVID Gene Functional Classification Tool: a novel biological modulecentric algorithm to functionally analyze large gene lists. Genome Biol. 2007;8(9):R183.

45. Betel D, Wilson M, Gabow A, Marks DS, Sander C. The microRNA.org resource: targets and expression. Nucleic Acids Res. 2008;36(Database issue): D149-53.

46. Chatr-Aryamontri A, Breitkreutz BJ, Oughtred R, Boucher L, Heinicke S, Chen D, et al. The BioGRID interaction database: 2015 update. Nucleic Acids Res. 2015:43(Database issue):D470-8.

47. Bindea G, Mlecnik B, Hackl H, Charoentong P, Tosolini M, Kirilovsky A, et al. ClueGO: a Cytoscape plug-in to decipher functionally grouped gene ontology and pathway annotation networks. Bioinformatics. 2009;25(8): 1091-3.

48. Swift F, Tovsrud N, Sjaastad I, Sejersted OM, Niggli E, Egger M. Functional coupling of alpha(2)-isoform $\mathrm{Na}(+) / \mathrm{K}(+)$-ATPase and $\mathrm{Ca}(2+)$ extrusion through the $\mathrm{Na}(+) / \mathrm{Ca}(2+)$-exchanger in cardiomyocytes. Cell Calcium. 2010; 48(1):54-60.

49. Anders $\mathrm{S}$, Huber W. Differential expression analysis for sequence count data. Genome Biol. 2010;11(10):R106.

50. Luxan G, Casanova JC, Martinez-Poveda B, Prados B, D'Amato G, MacGrogan D, et al. Mutations in the NOTCH pathway regulator MIB1 cause left ventricular noncompaction cardiomyopathy. Nat Med. 2013; 19(2):193-201.

51. Bullard JH, Purdom E, Hansen KD, Dudoit S. Evaluation of statistical methods for normalization and differential expression in mRNA-Seq experiments. BMC Bioinformatics. 2010;11.

52. Oshlack A, Wakefield MJ. Transcript length bias in RNA-seq data confounds systems biology. Biol Direct. 2009;4.

53. Roberts A, Trapnell C, Donaghey J, Rinn JL, Pachter L. Improving RNA-Seq expression estimates by correcting for fragment bias. Genome Biol. 2011; 12(3):R22. 
54. Benjamini Y, Hochberg Y. Controlling the False Discovery Rate - a Practical and Powerful Approach to Multiple Testing. J Roy Stat Soc B Met. 1995; 57(1):289-300.

55. Ritchie W, Flamant S, Rasko JE. Predicting microRNA targets and functions: traps for the unwary. Nat Methods. 2009;6(6):397-8.

56. lancu OD, Kawane S, Bottomly D, Searles R, Hitzemann R, McWeeney S. Utilizing RNA-Seq data for de novo coexpression network inference. Bioinformatics. 2012;28(12):1592-7.

57. David R, Groebner M, Franz WM. Magnetic cell sorting purification of differentiated embryonic stem cells stably expressing truncated human CD4 as surface marker. Stem Cells. 2005;23(4):477-82.

Submit your next manuscript to BioMed Central and we will help you at every step:

- We accept pre-submission inquiries

- Our selector tool helps you to find the most relevant journal

- We provide round the clock customer support

- Convenient online submission

- Thorough peer review

- Inclusion in PubMed and all major indexing services

- Maximum visibility for your research

Submit your manuscript at www.biomedcentral.com/submit
Biomed Central 